\title{
Is There a Best Symbolic Cycle-Detection Algorithm?
}

\author{
Kathi Fisler ${ }^{1,4}$, Ranan Fraer ${ }^{2}$, Gila Kamhi ${ }^{2}$, Moshe Y. Vardi ${ }^{1 \star}$, and Zijiang \\ Yang $^{1,3}$ \\ 1 Department of Computer Science, Rice University, Houston, TX, USA \\ 2 Intel Development Center, Haifa, Israel \\ 3 CCRL, NEC, Princeton, NJ, USA \\ ${ }^{4}$ Worcester Polytechnic Institute, Worcester, MA, USA
}

\begin{abstract}
Fair-cycle detection, a core problem in model checking, is solvable in linear time in the size of the design model using an explicitstate representation. Existing cycle-detection algorithms for symbolic model checking are quadratic or $n \log n$ time in the worst case and often inefficient in practice. Which default symbolic cycle-detection algorithm to implement in model checkers remains an open question. We compare several such algorithms based on the numbers of external and internal iterations and the numbers of image operations that they perform on both randomly-generated and real examples. Unlike recent work by Ravi, Bloem, and Somenzi, we conclude that model checkers need to implement at least two generic cycle-detection algorithms: the traditional EmersonLei algorithm and one that evolved from our study, originally due to Hojati et al. We demonstrate that these two algorithms are complementary, as the latter algorithm is provably incomparable to Emerson-Lei's and often dominates it in practice.
\end{abstract}

\section{Introduction}

Model checking, whether for LTL, CTL, or w-automata, has linear time complexity in the size of the design model. This well-known result follows from two facts: first, that most model checking techniques reduce to the problem of locating cycles through a given set of nodes in a graph $\mathbf{3} I 8$; second, that cycle detection is solvable in linear time using a depth-first search that identifies strongly-connected components ( $c f, 4)$. This depth-first strategy provides a suitable approach to cycle detection in explicit-state model checking, and has been implemented in several tools 711 .

Depth-first approaches to cycle detection are not suitable for BDD-based symbolic model checking because BDDs represent sets of states while depth-first search examines individual states. Efficient BDD-based model checking requires efficient breadth-first, set-based cycle-detection algorithms. Most modern symbolic model checkers employ some variant of Emerson and Lei's symbolic cycledetection algorithm 5. CTL model checkers use the Emerson-Lei algorithm

\footnotetext{
* Work partially supported by NSF Grant CCR-9988322 and a grant from the Intel corporation.
} 
(henceforth EL) to process formulas of the form $\mathrm{EG} \varphi$, which specify infinite paths on which every state satisfies $\varphi$. Linear-time model checkers compose the design model with an automaton representing the negation of the property, then check for cycles in the product automaton using the CTL formula EG true. Unfortunately, EL's time complexity is not linear in the size of the design model: the algorithm contains a doubly-nested fixpoint operator, and hence requires time quadratic in the design size in the worst case. The algorithm is also often slow in practice. EL is a so-called SCC-hull algorithm 16 . SCC-hull algorithms compute the set of states that contains all fair cycles. In contrast, SCC-enumeration algorithms enumerate all the strongly connected components of the state graph. While SCC-enumeration algorithms have a better worst-case complexity than SCC-hull algorithms 1, their performance in practice seems to be inferior to that of SCC-hull algorithms 16. This paper focuses on SCC-hull algorithms.

Researchers have proposed several alternatives to EL 81014 . Ravi, Bloem, and Somenzi have presented both a classification scheme for such algorithms and an experimental comparison of several algorithms with EL 16. They concluded that no algorithm consistently outperforms EL for cycle detection, and, consequently, there is no reason to "dethrone" EL as the default cycle-detection algorithm. Their comparison, however, is based primarily on running times, and secondarily on numbers of image operations. This approach has two significant drawbacks: it provides no useful feedback on why the algorithms behave as observed, and it suggests no techniques for predicting when one algorithm might outperform another. Furthermore, their comparison considers some algorithms that are based on post-image operations and some that are based on pre-image operations (as is EL), making it rather difficult to draw firm conclusions.

This paper demonstrates a methodology that both addresses these concerns and identifies a symbolic cycle-detection algorithm that provides a viable alternative to EL. Ravi et al. present bounds on the number of image operations performed by various cycle-detection algorithms. We argue that to understand the performance of SCC-hull algorithms one needs to measure both the number of image computations as well as the number of external iterations (defined in Section 2 . Our methodology focuses on the number of external iterations performed as a basis for comparing and refining symbolic cycle-detection algorithms. In aiming to balance the numbers of external and internal iterations performed, we have identified an algorithm that, as we argue, should join EL as a generic cycle-detection algorithm. We demonstrate that this algorithm is incomparable to EL, dominating it in many cases. Our conclusion is that, as in many other aspects of model checking, there is no "best" cycle-detection algorithm and model checkers need to implement at least both EL and our algorithm.

Section $\boldsymbol{\lambda}$ describes our analyses of three existing symbolic cycle-detection algorithms and shows how the competitive algorithm evolved from these analyses. Section $\mathbf{3}$ presents experimental results on randomly generated and real examples for both the special case of terminal and weak systems and more general examples. Section 4 compares the competitive algorithm to a specialized cycle-detection algorithm for terminal and weak systems. Section 5 concludes. 


\section{Symbolic Cycle-Detection Algorithms}

Cycle-detection algorithms in the context of model checking search for "bad" cycles in a directed graph representing a transition system modeling a design undergoing verification. Two parameters specify which cycles are considered bad: the invariant and the fair sets. The invariant specifies a condition, such as a propositional formula, that must be true of every state on a bad cycle. The fair sets specify sets of states that every bad cycle must pass through. We write $\mathrm{EG}_{\text {fair }} \varphi$ to indicate a search for cycles satisfying invariant $\varphi$ and passing through fair sets fair. We will omit the fair annotation when all states are considered fair.

Cycle detection in BDD-based model checking is challenging because the BDDs co-mingle information about different paths through a design. Symbolic cycle-detection algorithms maintain a set of states that may lead to bad cycles; this set is conservative, in that it contains all states that do lead to bad cycles. We call this the approximation set. The algorithms repeatedly refine the approximation set by locating and removing states that cannot lead to a bad cycle; we call this the pruning step. If a state lies on a bad cycle, then it must have a successor and a predecessor on that same cycle (and thus also in the approximation set). Cycle-detection algorithms use this information in different ways.

Formally, these algorithms search for cycles in nondeterministic transition systems. A transition system is a tuple $\left\langle Q, R, Q_{0}, \mathcal{F}\right\rangle$, where $Q$ is a set of states, $Q_{0} \subseteq Q$ is the initial state set, $R \subseteq Q \times Q$ is the transition relation, and $\mathcal{F} \subseteq Q$ is the set of fair states. A transition system is weak iff (1) there exists a partition of $Q$ into sets $Q_{1}, \ldots, Q_{n}$ such that each $Q_{i}$ is either contained in $\mathcal{F}$ or is disjoint from it, and (2) the $Q_{i}$ 's are partially ordered so that there is no transition from $Q_{i}$ to $Q_{j}$ unless $Q_{i} \leq Q_{j}$. If the $Q_{i}$ 's contained in $\mathcal{F}$ are the maximal elements of the partial order, a weak system is called terminal. This definition of weak and terminal transition systems is due to Bloem, Ravi, and Somenzi 2, as refined from Kupferman and Vardi 15. In model checking, designs commonly have several fair sets, and bad cycles must pass through each fair set. Such designs are outside the scope of weak systems, whose definition is only meaningful for one fair set 1

EL appears in Figure $\mathbf{I}$ (left) $\mathbf{2}$ At each iteration through the while loop, EL computes the set of states that can reach every fair set via a non-trivial path contained in the approximation set, $b$. We call these iterations external; the reachability computations (the EU formula) form the internal iterations. EL does most of its work in the internal iterations: each external iteration performs only one preimage computation per fair set outside of the internal iterations.

Hardin et al. attempted to reduce the number of external iterations that EL performs as a means of achieving an improved algorithm 8 . Their algorithm, called Catch-Them-Young (henceforth CTY), aggressively prunes the set

\footnotetext{
${ }^{1}$ LTL-to-automaton translation algorithms may yield multiple fair sets when one would suffice, rendering an otherwise weak system non-weak. Thus, minimizing the number of fair sets is an important optimization.

${ }^{2}$ Figure 11 shows VIS' implementation of EL; in SMV, the final image computation $(b:=b \wedge \mathrm{EX} d)$ is outside the for loop.
} 
and CTY is that EL prunes based only on successors, whereas CTY considers both successors and predecessors. An intermediate approach could perform CTY's repeated pruning, but using only pre-image computations, as in EL 19. This could greatly reduce the number of image computations of CTY, though perhaps at the expense of some additional external iterations. The resulting algorithm, called One-Way-Catch-Them-Young (henceforth owCTY), appears in Figure l (right) I oWCTY is essentially the pre-image version of Hojati et al.'s EL2 algorithm (sans an initial reachability computation) 10; its pruning strategy is similar in spirit to that of Kesten et al.'s algorithm for cycle detection in the presence of strong fairness 14 (which uses forward instead of backward image operations).

How do owCTY and EL compare? Hojati et al.'s experiments on a small set of small examples discussed only running time and were inconclusive for these two algorithms. Ravi et al.'s experiments compared EL and the forwardoperator version of EL2/OWCTY; this is not too meaningful, since the issue of forward vs. backward reachability 9 is orthogonal to the balance between external and internal iterations (indeed, the upper bounds obtained in 16 for EL and forward-EL 2 are incomparable). owCTY's worst-case running time has only a linear overhead (see below) over the $\mathcal{O}(|\mathcal{F}| d h)$ worst-case upper bound that Ravi et al. identified for EL 16 (where $|\mathcal{F}|$ is the number of fairness constraints, $d$ is the diameter of the state graph, and $h$ is the length of the longest reachable path in the SCC quotient graph). A worst-case analysis as done in 16 provides, however, only a very coarse comparison between the two algorithms. First, the overhead of owCTY over EL is not very significant. Second, the worst-case instances for EL may be different than those for OWCTY, which means that the comparison of worst-case running times does not tell us how the two algorithms compare on a given input instance. A more meaningful analysis would compare how the two algorithms perform on concrete instances. Analysis at this level shows that the two algorithms are incomparable. Figure 2 illustrates the differences between the EL and oWCTY pruning strategies; OWCTY outperforms EL on the first transition system, while EL outperforms owCTY on the second.

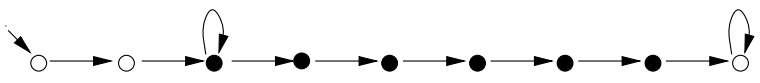

(2)

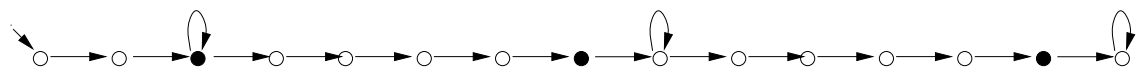

Fig. 2. Two transition systems that illustrate the differences between EL and owCTY. Black circles denote fair states. All states satisfy the invariant.

Consider the first transition system. Both algorithms eliminate the rightmost state in the first iteration and capture the remaining states in the approximation set. During the first iteration, owCTY eliminates all but the leftmost fair state;

${ }^{4}$ A variant of owCTY performs pruning inside the for loop; in practice, neither version consistently outperforms the other. 
EL eliminates only the rightmost fair state. EL requires an additional iteration to eliminate each of the four middle fair states. Each iteration involves a reachability computation that owCTY does not perform. If the chain of fair states in the first system contained $n$ fair states, OWCTY would perform $\mathcal{O}(n)$ image computations while EL would perform $\mathcal{O}\left(n^{2}\right)$ image computations. Thus, EL has a quadratic overhead relative to OWCTY on such systems.

Now consider the second transition system. In the first iteration, both algorithms eliminate the rightmost state and retain the remaining states in the approximation set. During the first iteration, EL throws away the rightmost fair state. The reachability computation in the second external iteration begins at the middle fair state; thus, EL eliminates the non-fair states between the right two fair states without traversing them explicitly again. owCTY, in contrast, uses an additional image computation to eliminate each of those non-fair states. The second system currently contains two copies of a chain of states consisting of four non-fair states, followed by a fair state, followed by a non-fair state with a self loop. If the system had $k$ consecutive copies of this chain, each with $m$ states in the initial non-fair chain, EL would perform $\mathcal{O}\left(k^{2} m\right)$ image computations as compared to oWCTY's $\mathcal{O}\left(k^{2} m+k m\right)=\mathcal{O}\left(k^{2} m\right)$ image computations. That is, the overhead of owCTY relative to EL is only linear.

In general, the two algorithms are incomparable with respect to their numbers of image computations. As oWCTY provably performs no more external iterations than EL, OWCTY's overhead (if it exists at all) is caused by the last line of the algorithm, which prunes the approximation set. Thus, owCTY's overhead is at most linear relative to EL, while, as we saw, EL can have a quadratic overhead relative to owCTY.

To gain a better picture on the comparative performance of EL, CTY, and owCTY, the experimental analyses in Section 3 gather data on the numbers of external iterations across several randomly generated and real examples; to complement the Ravi et al. study 16 , we also include running time, memory usage, and BDD size statistics. Our analyses show that owCTY requires almost the same number of external iterations as CTY with far fewer image computations; in practice, oWCTY almost always matches or improves on EL's performance.

\section{Comparative Analysis of the Algorithms}

\subsection{Experiments on Random Systems}

Our first set of experiments compares the algorithms on random systems. We generate random systems by generating random directed graphs. We would like to obtain directed graphs with non-uniform out-degree and linear density (i.e., a linear number of edges in the number of nodes); linear density prevents cycle detection from becoming trivial due to an excess or paucity of edges. The following model of random graphs, due to Karp 13, satisfies these criteria:

Definition 1 For each positive integer $n$ and each $p$ with $0<p<1$, the sample space consists of all labeled digraphs $D_{n, p}$ with $n$ vertices and edge probability $p$. 
Given a graph $G$ with vertices $V$ and edges $E$, the order of $G$ is $|V|$ and the density of $G$ is $|E| /|V|$. We will use $n$ and $d$ to represent a graph's order and density, respectively. We wish to generate graphs in the space $D_{n, d / n}$. Generating the graphs directly based on this model becomes time consuming as $n$ grows larger: the procedure must decide whether to include each of the possible $n(n-1)$ edges based on the probability $d / n$. Instead, we fix the number of edges to be the expected number $d n$, and choose $d n$ distinct edges from the $n(n-1)$ candidates. This approach provides a very good approximation to the given model 19 .

Our experiments compare four algorithms: EL, CTY, CTY + , and OWCTY. CTY + is a variant of CTY that restricts the reachability computations to consider only paths through the approximation set, rather than through the entire state space as in CTY 19; in other words, CTY + replaces line 5 of CTY with $b:=$ $\mathrm{E}\left[\begin{array}{lll}b & \mathrm{U} & \mathcal{F}_{i}\end{array}\right] \wedge \mathrm{E}\left[\begin{array}{lll}b & \mathrm{~S} & \mathcal{F}_{i}\end{array}\right]$, where $\mathrm{S}$ is the past-time operator since. We present two sets of results. The first measures the number of external iterations that each algorithm performs, the next measures the number of image computations that each algorithm performs $\$$ The experiments use graphs with order $2^{12}$ and densities varying over $1.2,1.6,2.0$, and 2.4 . This order is large enough to explore the behavior of the algorithms, yet small enough to analyze in a reasonable amount of time. We define a single fair set for each graph, with size varying over $.01 n, .1 n, .3 n, .5 n, .7 n$, and $.9 n$ where $n$ is the digraph order. Each experiment fixes either the density or the size of the fair set and varies the other. The figures reported in the rest of this section are averaged over 100 individual experiments.

\begin{tabular}{|l|c|c|c|c|}
\hline \multirow{2}{*}{} & \multicolumn{4}{|c|}{$|\mathcal{F}|$} \\
\cline { 2 - 5 } & $.01 n$ & $.1 n$ & $.5 n$ & $.9 n$ \\
\hline CTY & 2.18 & 2.41 & 2.09 & 2.00 \\
CTY+ & 2.18 & 2.41 & 2.09 & 2.00 \\
OWCTY & 2.17 & 2.37 & 2.07 & 2.00 \\
EL & 2.66 & 5.36 & 13.20 & 20.89 \\
\hline
\end{tabular}

\begin{tabular}{|l|c|c|c|c|}
\hline \multirow{2}{*}{} & \multicolumn{4}{|c|}{$d$} \\
\cline { 2 - 5 } & 1.2 & 1.6 & 2.0 & 2.4 \\
\hline CTY & 2.00 & 2.00 & 2.00 & 2.00 \\
CTY + & 2.00 & 2.00 & 2.00 & 2.00 \\
OWCTY & 2.00 & 2.00 & 2.00 & 2.00 \\
EL & 20.89 & 10.37 & 7.02 & 5.09 \\
\hline
\end{tabular}

Table 1. Average number of external iterations on digraphs with order $2^{12}$. The left table fixes the density at 1.2 and varies the fair set size. The right table fixes the fair set size at $.9 \times 2^{12}$ and varies the density.

Table Il shows the number of external iterations on digraphs with order $n=2^{12}$. One set of experiments fixes the density at 1.2 and varies the fair set size; the other fixes the fair set size at $.9 \times 2^{12}$ and varies the density. The tables indicate that CTY, CTY + and owCTY perform far fewer external iterations than EL. Furthermore, owCTY performs essentially the same number of external iterations as CTY; thus pruning based on predecessors as well as successors, as CTY does, does not significantly reduce the number of external iterations over a pruning strategy based only on successors. We therefore expect owCTY to consume considerably fewer resources than CTY in practice. EL requires significantly

${ }^{5}$ We refer to post- and pre-image computations collectively as image computations. 
more external iterations as the fair set grows larger, and significantly fewer external iterations as the density increases. In contrast, CTY, CTY + , and owCTY perform fairly consistent numbers of external iterations in both cases.

The data in Table $\boldsymbol{\|}$ do not indicate that CTY and owCTY are more efficient than EL because the former algorithms may do more work in the internal iterations. The number of image computations offers a more precise efficiency comparison. Image computations are the most computationally expensive operations in each of the cycle-detection algorithms. The cost of these operations depends on the density and order of the underlying graphs II. Since we analyze the four algorithms over the same randomly generated graphs, the cost of individual image computations is comparable across the algorithms. The number of image computations is therefore a fair parameter for comparing the algorithms.

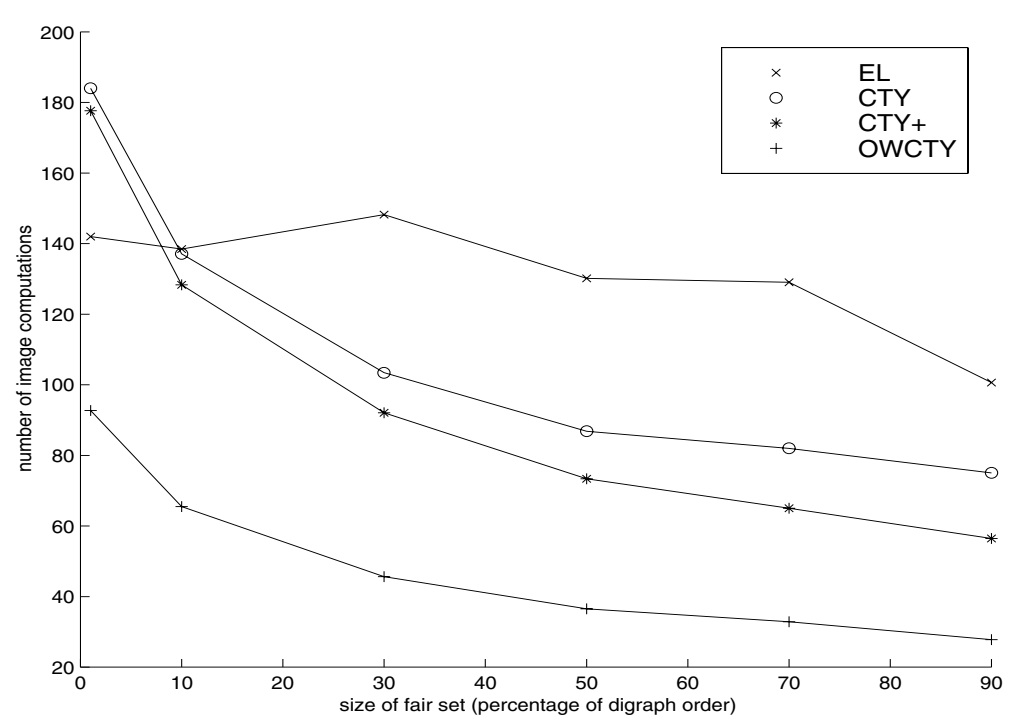

Fig. 3. Number of image computations for EL, CTY, CTY + and owCTY.

Figure $\$$ shows the number of image computations performed over graphs with order $n=2^{12}$, density $d=1.2$, and fair set size ranging over $.01 n, .1 n, .3 n$, $.5 n, .7 n$, and $.9 n$. For CTY, CTY + and owCTY the number of image computations decreases as the fair set gets larger. CTY performs more image computations than CTY + because CTY+ restricts reachability computations to the approximation set, which allows the computation to converge faster. owCTY performs fewer image computations than either CTY or CTY + because it does not perform forwards reachability. Separate data (not shown) show that the backwards reachability computations in OWCTY and CTY perform almost the same numbers of image computations; furthermore, the pruning step in OWCTY performs roughly half as many image computations as that in $\mathrm{CTY}+19$. Thus, eliminating 
the forward image computations makes OWCTY less computationally expensive without adversely affecting the number of external iterations required.

Separate experiments (not shown) show that the number of image computations decreases sharply as the density increases 19. In the case of EL, the number of image computations drops because the algorithm performs fewer external iterations as density increases, as discussed previously. For the remaining three algorithms, our experimental data shows that the size of the approximation set after each iteration becomes larger as the density increases. The approximation set determines the base set for subsequent reachability computations. The larger the base set, the faster reachability computations converge 19. Therefore, fewer image computations are needed when the digraph density increases. Although each pruning step removes fewer vertices, the final approximation set is also larger, so the algorithms perform fewer image computations as density increases. Plots for running time statistics are similar to those for image computations. In particular, both oWCTY and CTY consistently outperform EL. This contradicts the mixed results in other CTY versus EL experiments 816 .

\subsection{Experiments on Real Systems}

Our real design examples come from the VIS distribution and from Fabio Somenzi. They include an ethernet protocol with varying numbers of collisions before failure, a tree-structured arbiter with 8 nodes, a gcd circuit, a floating point multiplier, and two mutual exclusion protocols (bakery and eisenberg). These examples are written in Verilog and evaluated using the VIS model checker 17. We implemented owCTY within the VIS framework by replacing the original (EL) algorithm for evaluating EG formulas with owCTY in a copy of VIS. We ran the experiments using VIS version 1.3 (with version 1.2 of the vl2mv compiler), on an Intel 686 machine with $1 \mathrm{~GB}$ of memory running RedHat Linux version 2.2.12-20; our VIS installation uses the CUDD BDD package.

Table $\boldsymbol{z}$ summarizes experiments with LTL model checking of terminal and weak systems. For each LTL experiment, we evaluated $\mathrm{EG}_{\text {fair }}$ true on the product of the original design and a manually-constructed automaton for the negation of the property. Table $\mathbf{B}$ covers examples with multiple fair sets in the context of CTL model checking. Table 1 covers LTL model checking under multiple fairness constraints. In each table, stars on experiment names denote that the models contained cycles or that the property failed. The EX/EY and EU/ES figures count the number of image and reachability computations performed, respectively 6

The tables show that owCTY generally matches or outperforms EL, while CTY and CTY + are clearly not competitive. In many cases, oWCTY outperforms EL dramatically; in contrast, we have not yet found an example on which EL significantly outperforms OWCTY. The benefits of OWCTY are particularly evident on the ethernet and gcd examples in Table $\boldsymbol{\lambda}$ As expected, owCTY uses fewer external iterations than EL; however, oWCTY sometimes performs more image computations than EL.

\footnotetext{
${ }^{6}$ The EU/ES counts do not include trivial computations of the form $[\varphi \mathrm{U} \varphi]$.
} 


\begin{tabular}{|c|c|c|c|c|c|c|}
\hline Experiment & Procedure & $\begin{array}{l}\text { Ext. } \\
\text { Iter. }\end{array}$ & $\begin{array}{l}\text { EX or } \\
E X / E Y\end{array}$ & $\begin{array}{l}\text { Time } \\
\text { (sec) }\end{array}$ & $\begin{array}{l}\text { Mem } \\
\text { (MB) }\end{array}$ & $\begin{array}{c}\text { peak live } \\
\text { BDD nodes }\end{array}$ \\
\hline \multirow[t]{3}{*}{ ethernet 1} & EL & \begin{tabular}{|l|}
51 \\
\end{tabular} & 2179 & 356.6 & 13.6 & 339932 \\
\hline & CTY & 2 & $42 / 43$ & 187.9 & \begin{tabular}{|l|}
14.6 \\
\end{tabular} & 398280 \\
\hline & CTY+ & 2 & $41 / 42$ & 184.8 & 14.6 & 398280 \\
\hline $\mathrm{G}(p \rightarrow \mathrm{F} q)$ & OWCTY & 3 & 57 & 5.5 & 11.7 & 175118 \\
\hline \multirow[t]{3}{*}{ ethernet 2} & EL & 107 & 6506 & 10656.1 & \begin{tabular}{|l|}
14.4 \\
\end{tabular} & 367135 \\
\hline & $\overline{\text { CTY }}$ & 2 & $67 / 68$ & \begin{tabular}{|l|}
1893.6 \\
\end{tabular} & \begin{tabular}{|l|}
33.6 \\
\end{tabular} & 1365367 \\
\hline & CTY+ & 2 & $66 / 67$ & \begin{tabular}{|l|}
1887.6 \\
\end{tabular} & 33.6 & 1365755 \\
\hline $\mathrm{G}(p \rightarrow \mathrm{F} q)$ & OWCTY & 3 & 113 & 59.6 & \begin{tabular}{|l|}
14.1 \\
\end{tabular} & 404723 \\
\hline \multirow[t]{3}{*}{ ethernet 3} & EL & 171 & 11914 & \begin{tabular}{|l|}
4371.3 \\
\end{tabular} & \begin{tabular}{|l|}
13.7 \\
\end{tabular} & 279823 \\
\hline & $\overline{\text { CTY }}$ & 2 & $95 / 96$ & \begin{tabular}{|l|}
1962.2 \\
\end{tabular} & 35 & 1456597 \\
\hline & $\begin{array}{l}\text { CTY+ } \\
\text { CTH }\end{array}$ & 2 & $94 / 95$ & \begin{tabular}{|l|}
1938.0 \\
\end{tabular} & 35 & 1456597 \\
\hline $\mathrm{G}\left(p \rightarrow \mathrm{F}_{q}\right)$ & \begin{tabular}{|l} 
OWCTY \\
\end{tabular} & $\overline{3}$ & 177 & 24.6 & \begin{tabular}{|l|}
13.8 \\
\end{tabular} & 290593 \\
\hline \multirow[t]{3}{*}{ ethernet 4} & EL & - & - & $(30 \mathrm{H})$ & - & - \\
\hline & CTY & 2 & $130 / 131$ & \begin{tabular}{|l|}
5859.7 \\
\end{tabular} & \begin{tabular}{|l|}
53.6 \\
\end{tabular} & 2320201 \\
\hline & CTY+ & 2 & $130 / 2$ & 5895 & \begin{tabular}{|l|}
53.6 \\
\end{tabular} & 2320201 \\
\hline $\mathrm{G}\left(p \rightarrow \mathrm{F}_{q}\right)$ & OWCTY & 3 & 245 & 491.4 & 14.1 & 368225 \\
\hline \multirow[t]{3}{*}{ treearb $8^{*}$} & EL & 8 & 75 & 6.2 & 13.6 & 234021 \\
\hline & \begin{tabular}{|l|} 
CTY \\
\end{tabular} & - & - & \begin{tabular}{|l|}
$(20 M)$ \\
\end{tabular} & \begin{tabular}{|l}
$(23)$ \\
\end{tabular} & - \\
\hline & $\overline{\mathrm{CTY}+}$ & - & - & \begin{tabular}{|l|}
$(20 M)$ \\
\end{tabular} & \begin{tabular}{|l|}
$(23)$ \\
\end{tabular} & - \\
\hline \multirow{2}{*}{$\frac{\mathrm{G}\left(p \rightarrow \mathrm{F}_{q}\right)}{\mathrm{gcd}}$} & OWCTY & 2 & 24 & 4.2 & \begin{tabular}{|l|}
12.7 \\
\end{tabular} & 206640 \\
\hline & EL & - & - & $(37 \mathrm{H})$ & $\begin{array}{lll}- & \\
- & & l\end{array}$ & 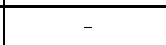 \\
\hline \multirow{2}{*}{ gcd } & $\overline{\text { CTY }}$ & 2 & $15 / 3$ & 1384.2 & \begin{tabular}{|l|}
59.3 \\
\end{tabular} & 2298351 \\
\hline & CTY+ & 2 & $14 / 2$ & 1383.0 & 59.3 & 2298351 \\
\hline $\mathrm{G}(p \rightarrow \mathrm{XF} q)$ & OWCTY & 2 & 24 & 2497.5 & 130.9 & 6285856 \\
\hline \multirow[t]{3}{*}{ fpmult } & EL & 2 & 18 & 18345.8 & 363 & 17667058 \\
\hline & $\overline{\text { CTY }}$ & 2 & $26 / 3$ & \begin{tabular}{|l|}
33089.7 \\
\end{tabular} & \begin{tabular}{|l|}
369 \\
\end{tabular} & 17619441 \\
\hline & $\begin{array}{l}\text { CTY+ } \\
\text { CTY }\end{array}$ & 2 & $18 / 2$ & \begin{tabular}{|l|}
21994.7 \\
\end{tabular} & \begin{tabular}{|l|}
368 \\
\end{tabular} & 17619441 \\
\hline $\mathrm{G}(p \rightarrow \mathrm{XXX} q)$ & OWCTY & 2 & 17 & 22457.2 & \begin{tabular}{|l|}
369 \\
\end{tabular} & 17422253 \\
\hline
\end{tabular}

Table 2. LTL model checking on weak and terminal systems. Parenthesized times indicate terminated computations; $M$ indicates minutes instead of seconds. 


\begin{tabular}{|c|c|c|c|c|c|c|c|}
\hline Experiment & Procedure & $\begin{array}{l}\text { Num } \\
\text { Fair }\end{array}$ & $\begin{array}{l}\text { Ext. } \\
\text { Iter. }\end{array}$ & $\begin{array}{c}\mathrm{EX} / \mathrm{EU} \text { or } \\
\mathrm{EX} / \mathrm{EY} / \mathrm{EU} / \mathrm{ES}\end{array}$ & $\begin{array}{l}\text { Time } \\
(\mathrm{sec})\end{array}$ & $\begin{array}{l}\text { Mem } \\
\text { (MB }\end{array}$ & $\begin{array}{c}\text { peak live } \\
\text { BDD nodes }\end{array}$ \\
\hline \multirow[t]{3}{*}{ bakery ${ }^{*}$} & $\mathrm{EL}$ & 6 & 18 & $554 / 91$ & 1.3 & 6.2 & 34447 \\
\hline & CTY & 6 & 11 & $1371 / 650 / 67 / 66$ & 10 & 13.3 & 176492 \\
\hline & CTY+ & 6 & 12 & $344 / 299 / 51 / 50$ & 7.0 & 13 & 182755 \\
\hline \multirow{2}{*}{$\mathrm{AG}\left(p \rightarrow \mathrm{AF}_{q}\right)$} & OWCTY & 6 & 18 & $516 / 75$ & 1.6 & 6.1 & 36962 \\
\hline & EL & 6 & 18 & $490 / 92$ & 1.3 & 6.0 & 29524 \\
\hline \multirow{2}{*}{ bakery2 } & CTY & 6 & 11 & $1239 / 614 / 67 / 66$ & $\overline{9.4}$ & 13.3 & 176492 \\
\hline & CTY+ & 6 & 11 & $282 / 246 / 47 / 46$ & 6.0 & 12.7 & 180657 \\
\hline $\mathrm{AG}(p \rightarrow \mathrm{AF} q)$ & OWCTY & 6 & 18 & $444 / 72$ & 1.4 & 5.8 & 28849 \\
\hline \multirow[t]{3}{*}{ treearb8* } & EL & 8 & 15 & $382 / 106$ & 14.8 & 13.6 & 328115 \\
\hline & CTY & 8 & - & - & $(194 \mathrm{M})$ & $(112)$ & - \\
\hline & CTY+ & 8 & - & - & $(170 \mathrm{M})$ & $(123)$ & - \\
\hline $\mathrm{AG}\left(p \rightarrow \mathrm{AF}_{q}\right)$ & OWCTY & $\overline{8}$ & 13 & $416 / 104$ & 13.1 & 13.4 & 309449 \\
\hline \multirow[t]{3}{*}{ eisenberg2 } & $\mathrm{EL}$ & 6 & 27 & $669 / 124$ & 1.6 & 5.5 & 17352 \\
\hline & CTY & 6 & \begin{tabular}{|l|l|}
23 \\
\end{tabular} & $2159 / 2031 / 139 / 138$ & 7.8 & 11.2 & 180311 \\
\hline & CTY+ & 6 & 16 & $252 / 506 / 56 / 55$ & 3.8 & 8.6 & 148353 \\
\hline $\mathrm{AG}(p \rightarrow \mathrm{AF} q)$ & OWCTY & 6 & 27 & $631 / 102$ & 1.4 & 5.4 & 18504 \\
\hline \multirow[t]{3}{*}{ elevator* } & EL & 8 & 12 & $849 / 97$ & 498.2 & 13.8 & 275914 \\
\hline & CTY & 8 & - & - & $(104 \mathrm{M})$ & $(38)$ & - \\
\hline & CTY+ & 8 & - & - & $(104 \mathrm{M})$ & $(43)$ & - \\
\hline $\mathrm{AG}(p \rightarrow \mathrm{AF} q$ & OWCTY & 8 & 12 & $861 / 79$ & 536.8 & 13.6 & 275914 \\
\hline
\end{tabular}

Table 3. CTL model checking on systems with multiple fairness constraints.

\begin{tabular}{|c|c|c|c|c|c|c|c|}
\hline Experiment & Procedure & \begin{tabular}{|l|} 
Num \\
Fair \\
\end{tabular} & $\begin{array}{l}\text { Ext. } \\
\text { Iter. }\end{array}$ & $\begin{array}{c}\mathrm{EX} / \mathrm{EU} \text { or } \\
\mathrm{EX} / \mathrm{EY} / \mathrm{EU} / \mathrm{ES}\end{array}$ & $\begin{array}{l}\text { Time } \\
(\mathrm{sec})\end{array}$ & $\begin{array}{l}\text { Mem } \\
(\mathrm{MB}) \\
\end{array}$ & \begin{tabular}{|c|} 
peak live \\
BDD nodes
\end{tabular} \\
\hline \multirow[t]{3}{*}{ treearb8* } & EL & 9 & 15 & $1021 / 135$ & 1397.8 & 13.8 & 239731 \\
\hline & $\overline{\text { CTY }}$ & $\overline{9}$ & - & - & $(186 \mathrm{M})$ & $(44)$ & - \\
\hline & CTY+ & $\overline{9}$ & - & - & $(207 \mathrm{M})$ & $(157)$ & - \\
\hline \multirow{4}{*}{\begin{tabular}{|l}
$\mathrm{G}(p \rightarrow \mathrm{F} q)$ \\
eisenberg2
\end{tabular}} & OWCTY & $\overline{9}$ & 14 & $1000 / 126$ & 911.6 & 13.9 & 369062 \\
\hline & EL & 7 & 24 & $1332 / 161$ & 5.7 & 7.2 & 47704 \\
\hline & CTY & 7 & 24 & $5114 / 5486 / 169 / 168$ & 60.3 & 13.7 & 240028 \\
\hline & CTY+ & 7 & 15 & $229 / 399 / 53 / 52$ & 4.7 & 8.8 & 147763 \\
\hline$\frac{\mathrm{G}(p \rightarrow \mathrm{F} q)}{\text { elevator } 3^{*}}$ & OWCTY & 7 & 24 & $1197 / 109$ & 5.3 & 7.1 & 59802 \\
\hline \multirow[t]{3}{*}{ elevator $3^{*}$} & EL & 3 & 2 & $7 / 1$ & 1164.7 & 87.5 & 4062730 \\
\hline & CTY & - & - & - & $(60 \mathrm{M})$ & $(270)$ & $=$ \\
\hline & CTY+ & - & - & - & \begin{tabular}{|l|}
$(60 \mathrm{M})$ \\
\end{tabular} & $(270)$ & - \\
\hline $\mathrm{G} p$ & OWCTY & 3 & 2 & $13 / 1$ & 1167.3 & 87.5 & 4062730 \\
\hline \multirow[t]{4}{*}{ elevator $4^{*}$} & EL & 1 & 2 & $3 / 1$ & 16192.4 & 282 & 13308496 \\
\hline & $\overline{\text { CTY }}$ & 1 & - & - & $(365 \mathrm{M})$ & $(278)$ & - \\
\hline & CTY+ & 1 & - & - & $(367 \mathrm{M})$ & $(278)$ & - \\
\hline & OWCTY & 1 & 2 & $5 / 1$ & 16388.0 & 282 & 13308496 \\
\hline
\end{tabular}

Table 4. LTL model checking on systems with multiple fairness constraints. 


\begin{tabular}{|l|l|c|c|c|c|}
\hline Exp. & Proc. & $\begin{array}{c}\text { Num } \\
\text { Fair }\end{array}$ & $\begin{array}{c}\text { Ext. } \\
\text { Iter. }\end{array}$ & EX & $\begin{array}{r}\text { Time } \\
\text { (sec) }\end{array}$ \\
\hline A $^{*}$ & EL & 2 & 6 & 203 & 65.49 \\
\cline { 2 - 6 } & OWCTY & 2 & 2 & 77 & 32.58 \\
\hline \multirow{2}{*}{${ }^{*}$} & EL & 6 & 2 & 147 & 16.26 \\
\cline { 2 - 6 } & OWCTY & 6 & 2 & 149 & 16.33 \\
\hline E* $^{*}$ & EL & 4 & 3 & 125 & 6.89 \\
\cline { 2 - 6 } & OWCTY & 4 & 2 & 87 & 6.39 \\
\hline F* $^{*}$ & EL & 2 & 10 & 50 & 870.0 \\
\cline { 2 - 6 } & OWCTY & 2 & 2 & 27 & 897.7 \\
\hline H1 $^{*}$ & EL & 2 & 8 & 40 & 633.8 \\
\cline { 2 - 6 } & OWCTY & 2 & 2 & 23 & 495.7 \\
\hline H3 $^{*}$ & EL & 2 & 8 & 40 & 550.5 \\
\cline { 2 - 6 } & OWCTY & 2 & 2 & 23 & 592.7 \\
\hline
\end{tabular}

\begin{tabular}{|l|l|c|c|c|c|}
\hline Exp. & Proc. & $\begin{array}{c}\text { Num } \\
\text { Fair }\end{array}$ & $\begin{array}{c}\text { Ext. } \\
\text { Iter. }\end{array}$ & EX & $\begin{array}{c}\text { Time } \\
(\mathrm{sec})\end{array}$ \\
\hline \multirow{2}{*}{${ }^{*}$} & EL & 2 & 2 & 40 & 1004.5 \\
\cline { 2 - 6 } & OWCTY & 2 & 2 & 23 & 692.9 \\
\hline \multirow{3}{*}{$1^{*}$} & EL & 2 & 8 & 40 & 521.9 \\
\cline { 2 - 6 } & OWCTY & 2 & 2 & 23 & 426.6 \\
\hline \multirow{2}{*}{$2^{*}$} & EL & 2 & 8 & 40 & 447.9 \\
\cline { 2 - 6 } & OWCTY & 2 & 2 & 23 & 347.7 \\
\hline \multirow{2}{*}{${ }^{*}$} & EL & 2 & 7 & 25 & 220.3 \\
\cline { 2 - 6 } & OWCTY & 2 & 2 & 20 & 165.3 \\
\hline \multirow{2}{*}{ LW $^{*}$} & EL & 2 & 6 & 24 & 129.4 \\
\cline { 2 - 6 } & OWCTY & 2 & 2 & 19 & 129.4 \\
\hline \multirow{2}{*}{ M1* } & EL & 2 & 7 & 35 & 81.5 \\
\cline { 2 - 6 } & OWCTY & 2 & 2 & 21 & 53.9 \\
\hline \multirow{2}{*}{} & & & &
\end{tabular}

Table 5. Results from Intel on checking $\mathrm{EG}_{\text {fair }}$ true on systems that have (and require) multiple fairness constraints.

Finally, we compared owcTY and EL on Intel designs using internal Intel tools (Table 5. All the table entries reflect the composition of actual designs with linear-time properties, using multiple fairness constraints. oWCTY performed significantly better than EL in all examples except F and H3, where EL slightly outperformed owCTY.

\section{OWCTY Versus Specialized Algorithms}

Our experimental results show that owCTY generally outperforms EL on terminal and weak systems. Bloem, Ravi, and Somenzi have presented an algorithm that is specialized to verify terminal and weak systems efficiently 2 . Linear-time model checkers detect bad cycles by using the EL algorithm to check EG true over the product of the design and the negation of the desired property. Bloem et al. observed that for terminal and weak systems, CTL formulas capture the search for bad cycles. Specifically, the formulas EF fair and EF EG fair are true of terminal and weak systems, respectively, when they contain infinite fair cycles. Accordingly, their algorithm (henceforth BRs) checks one of the formulas EF fair, EF EG fair, or $\mathrm{EG}_{\text {fair }}$ true based on the structure of the input system. This structure follows from the structure of the property being tested: if a property corresponds to a weak (resp. terminal) system, the product of that property and a design model is also a weak (resp. terminal) system. Bloem et al. showed that BRS significantly outperforms EL in practice on terminal and weak systems.

Table 6 compares owCTY to BRS $\mathbf{q}$ For the examples from Table $\mathbf{2}$ we checked both $\mathrm{EG}_{\text {fair }}$ true and the appropriate formula from BRS using OWCTY. The statis-

\footnotetext{
7 The gcd and fpmult examples are the same as Bloem et al. used in their paper 2 . Our resource usage on these examples differs widely from theirs due to differences between our two versions of the compiler from Verilog to BLIF, the VIS input language.
} 


\begin{tabular}{|c|c|c|c|c|c|}
\hline Experiment & Procedure & EX & $\begin{array}{l}\text { Time } \\
\text { (sec) }\end{array}$ & $\begin{array}{l}\text { Mem } \\
\text { (MB }\end{array}$ & $\begin{array}{c}\text { peak } \\
\text { BDD nodes }\end{array}$ \\
\hline ethernet 1 & $\neg$ EF EG fair & 53 & 4.2 & 11.2 & 151306 \\
\hline $\mathrm{G}(p \rightarrow \mathrm{F} q)$ & EG $_{\text {fair }}$ true(oWCTY & 57 & 5.5 & 11.7 & 175118 \\
\hline ethernet 2 & $\neg$ EF EG fair & 109 & 24.4 & 13.7 & 381839 \\
\hline $\mathrm{G}(p \rightarrow \mathrm{F} q)$ & EG fair $_{\text {frue(owCTY }}$ & 113 & 59.6 & 14.1 & 404723 \\
\hline ethernet 3 & $\neg$ EF EG fair & 173 & 13.3 & 13.6 & 287787 \\
\hline $\mathrm{G}\left(p \rightarrow \mathrm{F}_{q}\right)$ & $\mathrm{EG}_{\text {fair }}$ true(owCTY & 177 & 24.6 & 13.8 & 290593 \\
\hline ethernet 4 & $\neg \mathrm{EF}$ EG fair & 241 & 145.6 & 14.0 & 373531 \\
\hline $\mathrm{G}(p \rightarrow \mathrm{F} q)$ & $\mathrm{EG}_{\text {fair }} \operatorname{true}(\mathrm{OWCTY}$ & 245 & 491.4 & 14.1 & 368225 \\
\hline treearb $8^{*}$ & $\neg$ EF EG fair & 22 & 4.1 & 12.6 & 200529 \\
\hline $\mathrm{G}(p \rightarrow \mathrm{F} q)$ & EG fairtrue(owCTY & 24 & 4.2 & 12.7 & 206640 \\
\hline & $\neg E F$ EG fair & 20 & 3351.6 & 193 & 8204281 \\
\hline $\mathrm{G}(p \rightarrow \mathrm{XF} q)$ & EG $_{\text {fairtrue(oWCTY }}$ & 24 & 2497.5 & 130.9 & 6285856 \\
\hline fpmult & $\neg \mathrm{EF}$ fair & 8 & 5565.5 & 329 & 16109729 \\
\hline $\mathrm{G}\left(p \rightarrow \mathrm{XXX}_{q}\right)$ & $\mathrm{EG}_{\text {fair }}$ true(owCTY & 17 & 22457.2 & 369 & 17422253 \\
\hline
\end{tabular}

Table 6. Comparison between the oWCTY and BRs algorithms.

tics on $\mathrm{EG}_{\text {fair }}$ true are reproduced from Table $\mathbf{2}$ The specialized approach outperforms owCTY on most of these examples (except the gcd example). This is due to the difference between checking $\mathrm{EG}_{\text {true }}$ fair (BRS) and $\mathrm{EG}_{\text {fair }}$ true (owCTY). The former restricts the search for a bad cycle to the fair states; the latter looks for a cycle that intersects the fair states. As a result, both EL and owCTY can have non-fair states in their approximation sets, while BRS' approximation set contains only fair states. This restriction usually allows BRS to converge faster.

This comparison demonstrates how exploiting structural information about systems can lead to more efficient verification algorithms. Note, however, that BRS is not a generic cycle-detection algorithm. Furthermore, we must also consider the cost of determining whether a system is weak or terminal, which is not included in our paper or in Bloem et al.'s. In theory, this operation can be done symbolically in $\mathcal{O}(n \log n)$ time I , but experimental results are not yet available. For the simple properties considered by Bloem et al. and here, this overhead is insignificant; for more complicated properties (such as those including complex environmental assumptions) it could be rather substantial. owCTY, which is a generic algorithm, performs well in practice without the overhead of specialized analyses as required in BRs.

\section{Conclusions}

Symbolic model checking remains a heuristic process, as metrics do not yet exist to predict BDD behavior under differing algorithms. As a result, comparative analyses of algorithms are extremely useful in helping tool developers choose which algorithms to implement. In the name of good science, these analyses need 
to be reproducible and portable to the greatest extent possible. Such analyses provide not only firm data, but a foundation for future algorithm development.

This paper compares three symbolic cycle-detection algorithms (and a variant on one of them) based on the number of iterations they take through their outermost fixpoint operator, as well as the number of image operations they perform. Each algorithm employs a slightly different strategy for pruning the set of states potentially lying on cycles. Our analysis shows that the original Emerson-Lei (EL) algorithm 5 performs too little work outside of its internal iterations, while Hardin et al.'s Catch-Them-Young (CTY) algorithm 8 performs too much. In contrast, Hojati's EL2 algorithm 10, which we view as a one-way version of CTY (OWCTY) does seem to balance the work inside and outside the internal iterations. On random examples and on terminal and weak systems, oWCTY dominates EL, while on general systems, OWCTY is competitive with EL, dominating it significantly in many cases. We have also shown that the two algorithms are incomparable with respect to the number of image computations they perform: EL can have a quadratic overhead over owCTY, while owCTY can have a linear overhead over EL. These results support our conclusion that model checkers need to contain both EL and owCTY.

In the course of this project, we have identified two desired features for verification tools. First, we want tools to implement multiple algorithms for common problems such as cycle-detection. Both our analysis and the recent one by Ravi et al. 16 indicate that no algorithm consistently outperforms the others; indeed, verification tasks may be tractable with one algorithm and intractable with another. Tools providing multiple algorithms afford human verifiers opportunities to experiment and find algorithms that work on their applications. A similar conclusion in the context of semi-exhaustive reachability analysis was reached in 6. Second, we want tools to provide visualizations of computational patterns during model checking. Intel's Palette 12 does some of this; we wish we had such a tool to augment VIS and other publicly-available tools. Testbeds supporting multiple algorithms and better data collection would provide strong support for more disciplined approaches to algorithm comparisons in verification.

\section{Acknowledgements}

We thank Kavita Ravi, Fabio Somenzi, and Roderick Bloem for their very helpful comments on this paper, and the Rice PLT group for access to their largememory server.

\section{References}

1. Bloem, R., H. N. Gabow and F. Somenzi. An algorithm for strongly connected component analysis in $n \log n$ symbolic steps. In Intl. Conf. on Formal Methods in Computer-Aided Verification, Lecture Notes in Computer Science. Springer-Verlag, 2000 . 
2. Bloem, R., K. Ravi and F. Somenzi. Efficient decision procedures for model checking of linear time logic properties. In Intl. Conf. on Computer-Aided Verification, Lecture Notes in Computer Science, pages 222-235. Springer-Verlag, 1999.

3. Clarke, E. M., E. A. Emerson and A. P. Sistla. Automatic verification of finitestate concurrent systems using temporal logic specifications. ACM Transactions on Programming Languages and Systems, 8(2):244-263, January 1986.

4. Courcoubetis, C., M. Y. Vardi, P. Wolper and M. Yannakakis. Memory efficient algorithms for the verification of temporal properties. Formal Methods in System Design, 1:275-288, 1992.

5. Emerson, E. A. and C. L. Lei. Efficient model checking in fragments of the propositional model mu-calculus. Proceedings of LICS 1986, pages 267-278, 1986.

6. Fraer, R., G. Kamhi, L. Fix and M. Y. Vardi. Evaluating semi-exhausting verification techniques for bug hunting. In Proceedings of the 1st Intl. Workshop on Symbolic Model Checking. Electronic Notes in Theoretical Computer Science, 1999.

7. Hardin, R. H., Z. Har'El and R. P. Kurshan. COSPAN. In Intl. Conf. on ComputerAided Verification, number 1102 in Lecture Notes in Computer Science, pages 423427. Springer-Verlag, 1996.

8. Hardin, R. H., R. P. Kurshan, S. K. Shukla and M. Y. Vardi. A new heuristic for bad cycle detection using BDDs. In Proc. Conf. on Computer-Aided verification (CAV'97), pages 268-278. Springer-Verlag. LNCS 1254, 1997.

9. Henzinger, T., O. Kupferman and S. Qadeer. From prehistoric to postmodern symbolic model checking. In Hu, A. and M. Vardi, editors, Intl. Conf. on ComputerAided Verification, volume 1427 of Lecture Notes in Computer Science, pages 195206. Springer-Verlag, 1998.

10. Hojati, R., H. Touati, R. Kurshan and R. Brayton. Efficient $\omega$-regular language containment. In Intl. Conf. on Computer-Aided Verification, number 663 in Lecture Notes in Computer Science. Springer-Verlag, 1992.

11. Holzmann, G. and D. Peled. The state of SPIN. In Intl. Conf. on Computer-Aided Verification, number 1102 in Lecture Notes in Computer Science, pages 385-389. Springer-Verlag, 1996.

12. Kamhi, G., L. Fix and Z. Binyamini. Symbolic model checking visualization. In Intl. Conf. on Formal Methods in Computer-Aided Verification, number 1522 in Lecture Notes in Computer Science, pages 290-303. Springer-Verlag, 1998.

13. Karp, R. M. The transitive closure of a random digraph. Random Structures and Algorithms, 1(1), 1990.

14. Kesten, Y., A. Pnueli and L. on Raviv. Algorithmic verification of linear temporal logic specifications. In Intl. Colloquium on Automata, Languages, and Programming, number 1443 in Lecture Notes in Computer Science. Springer-Verlag, 1998.

15. Kupferman, O. and M. Y. Vardi. Freedom, weakness, and determinism: From linear-time to branching-time. In IEEE Symp on Logic in Computer Science, 1998.

16. Ravi, K., R. Bloem and F. Somenzi. A comparative study of symbolic algorithms for the computation of fair cycles. In Intl. Conf. on Formal Methods in ComputerAided Verification, Lecture Notes in Computer Science. Springer-Verlag, 2000.

17. The VIS Group. VIS: A system for verification and synthesis. In Alur, R. and T. Henzinger, editors, Intl. Conf. on Computer-Aided Verification, volume 1102 of Lecture Notes in Computer Science. Springer-Verlag, July 1996.

18. Vardi, M. Y. and P. Wolper. An automata-theoretic approach to automatic program verification. In IEEE Symposium on Logic in Computer Science, 1986.

19. Yang, Z. Performance analysis of symbolic reachability algorithms in model checking. Master's thesis, Rice University, Department of Computer Science, 1999. Available at http://www.cs.rice.edu/CS/Verification/. 